\title{
Numerical Modeling of Stokes Flow in a Circular Cavity by Variational Multiscale Element Free Galerkin Method
}

\author{
Ping Zhang and Xiaohua Zhang \\ College of Science, China Three Gorges University, Yichang 443002, China \\ Correspondence should be addressed to Xiaohua Zhang; zhangxiaohua07@163.com
}

Received 31 May 2014; Accepted 21 July 2014; Published 14 August 2014

Academic Editor: Alex Elías-Zúñiga

Copyright (C) 2014 P. Zhang and X. Zhang. This is an open access article distributed under the Creative Commons Attribution License, which permits unrestricted use, distribution, and reproduction in any medium, provided the original work is properly cited.

\begin{abstract}
The variational multiscale element free Galerkin method is extended to simulate the Stokes flow problems in a circular cavity as an irregular geometry. The method is combined with Hughes's variational multiscale formulation and element free Galerkin method; thus it inherits the advantages of variational multiscale and meshless methods. Meanwhile, a simple technique is adopted to impose the essential boundary conditions which makes it easy to solve problems with complex area. Finally, two examples are solved and good results are obtained as compared with solutions of analytical and numerical methods, which demonstrates that the proposed method is an attractive approach for solving incompressible fluid flow problems in terms of accuracy and stability, even for complex irregular boundaries.
\end{abstract}

\section{Introduction}

The Stokes flow can be considered for a range of engineering processed and natural phenomena, and numerical solution of the Stokes equations for incompressible viscous fluids has been usually dominated by meshbased methods such as finite difference method (FDM), finite volume method (FVM), and finite element method (FEM). Although these methods have been successfully applied to computational fluid dynamics problems, their accuracy depends critically on mesh quality and they have many difficulties in dealing with some complex fluid flow problems. These difficulties can be overcome by meshless of mesh-free methods, which have attracted considerable interest over the past few years. Several review articles $[1,2]$ and books $[3-5]$ which cover the classification, application, and computer implementation of meshless methods have been reported. In recent years, the meshless methods have become an extensive area of research for solving fluid dynamics problems.

It is well known that the main issues germane to the development of a successful solver for incompressible Stokes flow are proper treatments of incompressibility. Improper treatments may result in spurious oscillations for velocity or pressure solutions. For meshless methods based on Galerkin formulations, the same problems will arise from the treatment of incompressibility constraint [6]; that is, they must either satisfy or avoid the Babuskka-Brezzi condition. So far, a number of stabilization methods have been developed to overcome numerical problems for meshless methods. The ways to deal with the Babuskka-Brezzi condition for most of meshless methods based on Galerkin weak from are used with the ideas of finite element method (FEM) to solve such problems, such as streamline-upwind Petrov-Galerkin [7, 8], pressure-stabilizing Petrov-Galerkin (PSPG) $[9,10]$, Galerkin least-squares (GLS) [11, 12], projection or fractional step method $[13,14]$ (e.g., characteristic-based split algorithm), and finite calculus approach (FIC) $[15,16]$. However, these methods are related to a stabilization parameter and it depends on the problem under consideration and the chosen numerical method. A detailed overview of stabilization approaches for meshless methods can refer to [17].

In the mid-1990s Hughes revisited the origins of the stabilization methods from a variational multiscale viewpoint and presented the variational multiscale method. In this method different stabilization methods appear as special cases of the underlying subgrid modeling concept [18]. Compared with other stabilization methods, the Hughes' variational multiscale method can get stabilization parameter 
naturally and allow the equal order interpolation for the velocity and pressure which is convenient for programming. In order to take advantage of meshless method and variational multiscale methods, Zhang et al. combined the meshless method and variational multiscale method which is called variational multiscale element free Galerkin method (VMEFG) for Stokes problems [19], water wave problems [20], and magnetohydrodynamics flow problems [21]. The VMEFG method allows equal order interpolations for the velocity and the pressure field, which are easy to implement but can violate the celebrated Babuskka-Brezzi condition. Meanwhile, the structure of the stabilization terms is derived consistently and the definition of the stabilization tensor is obtained naturally via the solution of the fine-scale problem. In this paper VMEFG method is extended to simulation of Stokes flow with complex domain, such as in a circular cavity. Study of slow and viscous flow in a cavity with rotating boundaries provides useful information on flow behavior and distributive mixing, which has many industrial applications [22].

An outline of the paper is as follows. In Section 2, the fundamental principle of EFG method is briefly described. Then, the VMEFG method for the Stokes fluid flow is expressed. In Section 4, numerical examples are presented to demonstrate the accuracy of our method. The paper ends with concluding remarks in Section 5.

\section{Review on the Element Free Galerkin Method}

In the EFG method, the field variable $u(\mathbf{x})$ is approximated by moving least squares (MLS) approximation, which consists of three parts: a basis function, a group of nonconstant coefficients, and a weight function associated with each node. According to the MLS approximation, the unknown function $u(\mathbf{x})$ can be written as follows [3]:

$$
u(\mathbf{x}) \approx u^{h}(\mathbf{x})=\sum_{j=1}^{m} p_{j}(\mathbf{x}) a_{j}(\mathbf{x})=\mathbf{P}^{T}(\mathbf{x}) \mathbf{a}(\mathbf{x}),
$$

where $\mathbf{P}(\mathbf{x})$ is a complete polynomial basis of order $m$ and $\mathbf{a}(\mathbf{x})$ is a vector of coefficients (to be determined) which is a function of the space coordinate $\mathbf{x}$. In the paper, we choose linear basis to approximate velocity and pressure.

Assume that we have known the nodal value $u_{i}=u\left(\mathbf{x}_{i}\right)$ for the function $u(\mathbf{x})$ at $n$ nodes in the domain $\Omega$. Then the unknown vector $\mathbf{a}(\mathbf{x})$ in (1) can be obtained at any point $\mathbf{x}$ by minimizing the following weighted, discrete error norm:

$$
\mathbf{J}=\sum_{i=1}^{n} w\left(\mathbf{x}-\mathbf{x}_{i}\right)\left[u_{i}-\mathbf{P}^{T}\left(\mathbf{x}_{i}\right) \mathbf{a}(\mathbf{x})\right]^{2} .
$$

Here $n$ is the number of nodes in the support domain of $\mathbf{x}$ in which the weight function $w\left(\mathbf{x}-\mathbf{x}_{i}\right)>0$. Usually, $w\left(\mathbf{x}-\mathbf{x}_{i}\right)$ are functions of a distant parameter $r_{i}=\left\|\mathbf{x}-\mathbf{x}_{i}\right\| / d_{i}$ where $d_{i}$ denotes the influence domain for node $\mathbf{x}_{i}$. Generally, $d_{i}=\alpha \rho_{i}$ where $\alpha$ is the dimensionless size of influence domain and $\rho_{i}$ is the distance between two adjacent nodes. The influence domain defines the degree of continuity between the nodes and the bandwidth of the system matrices; thus, it is crucial to solution accuracy and computational cost.

Minimization of (2) with respect to $\mathbf{a}(\mathbf{x})$ then yields the following system of linear equations for the vector $\mathbf{a}(\mathbf{x})$ :

$$
\mathbf{A}(\mathbf{x}) \mathbf{a}(\mathbf{x})=\mathbf{B}(\mathbf{x}) \mathbf{u},
$$

where $\mathbf{u}$ is the vector of nodal unknowns; matrices $\mathbf{A}(\mathbf{x})$ and $\mathbf{B}(\mathbf{x})$ are defined as

$$
\begin{gathered}
\mathbf{A}(\mathbf{x})=\sum_{i=1}^{n} w_{i}(\mathbf{x}) \mathbf{P}\left(\mathbf{x}_{i}\right) \mathbf{P}^{T}\left(\mathbf{x}_{i}\right), \\
\mathbf{B}(\mathbf{x})=\left[w_{1}(\mathbf{x}) \mathbf{P}\left(\mathbf{x}_{1}\right), w_{2}(\mathbf{x}) \mathbf{P}\left(\mathbf{x}_{2}\right), \ldots, w_{n}(\mathbf{x}) \mathbf{P}\left(\mathbf{x}_{n}\right)\right] .
\end{gathered}
$$

If $\mathbf{A}$ is invertible, solving (3), and then substituting $\mathbf{a}(\mathbf{x})$ back into (1), the MLS approximant can be defined as

$$
u^{h}(\mathbf{x})=\mathbf{P}^{T}(\mathbf{x}) \mathbf{A}^{-1}(\mathbf{x}) \mathbf{B}(\mathbf{x}) \mathbf{u}=\mathbf{N}^{T}(\mathbf{x}) \mathbf{u},
$$

where $\mathbf{N}(\mathbf{x})$ is the vector of MLS shape functions.

In general, the use of the MLS approximation produces shape functions that do not possess the Kronecker Delta condition property; that is, $\mathbf{N}_{i}\left(\mathbf{x}_{j}\right) \neq \delta_{i j}$. Thus, essential boundary conditions cannot be imposed as easily as that in the standard FEM. Until now, the implementation of essential boundary conditions is still an open research topic for meshless methods $[23,24]$. In the paper, a simple technique proposed by Zhang et al. [20] is utilized; that is, the nodal influence domain of meshless method is extended to have arbitrary polygon shape. This technique makes MLS approximation function possess interpolation property as $\alpha$ approach to 1 [25] and is easier to solve problems with complex area.

\section{The VMEFG Method for Stokes Problem}

3.1. The Stokes Problem and Its Standard Weak Form. The steady-state problem of a Newtonian, viscous, and incompressible fluid is governed by the Stokes equations:

$$
\begin{gathered}
-\eta \Delta \mathbf{u}+\nabla p=\mathbf{b} \quad \text { in } \Omega \\
\nabla \cdot u=0 \quad \text { in } \Omega,
\end{gathered}
$$

where the symbols $\Delta, \nabla$, and $\nabla \cdot$ represent the Laplacian, gradient, and divergence operators, respectively; $\mathbf{u}$ is the velocity vector; $p$ is the kinematic pressure; $\eta$ is the kinematic viscosity; and $\mathbf{b}$ is the body force per unit mass. For the sake of simplicity, the Stokes equations are subject to the no-slip boundary condition

$$
\mathbf{u}=\overline{\mathbf{u}} \text { on } \Gamma
$$

Let $\mathbf{w}$ and $q$ represent the weight functions for velocity $\mathbf{u}$ and pressure $p$, respectively; then based on the Galerkin 
formulation, the standard weak form of the problem (6) is given as follows:

$$
\begin{gathered}
(\nabla \mathbf{w}, \eta \nabla \mathbf{u})-(\nabla \cdot \mathbf{w}, p)=(\mathbf{w}, \mathbf{b}), \\
(q, \nabla \cdot \mathbf{u})=0,
\end{gathered}
$$

where $(\cdot, \cdot)=\int_{\Omega}(\cdot) d \Omega$.

The VMEFG method essentially contains three steps for the simulation of Stokes fluid flow. In the first step, the velocity field is decomposed into coarse scale and fine scale. In the second step, it uses bubble function to determine fine scale solution analytically. In the third step, it substitutes the fine scale solution into coarse scale problem and then obtains the coarse scale solution numerically. In the following, a brief introduction of VMEFG is presented and more details about the VMEFG can refer to $[19,20]$.

3.2. The Decomposition of the Standard Weak Form. Assume that the velocity $\mathbf{u}$ and its weight function $\mathbf{w}$ can be decomposed into coarse scale and fine scale, respectively; namely,

$$
\begin{aligned}
\mathbf{u} & =\overline{\mathbf{u}}+\widehat{\mathbf{u}}, \\
\mathbf{w} & =\overline{\mathbf{w}}+\widehat{\mathbf{w}},
\end{aligned}
$$

where $\overline{\mathbf{u}}$ and $\widehat{\mathbf{u}}$ are the coarse scale and fine scale for velocity $\mathbf{u}$ and $\overline{\mathbf{w}}$ and $\widehat{\mathbf{w}}$ are the coarse scale and fine scale for weight function $\mathbf{w}$, respectively. Meanwhile, we assume there exists a linearity between coarse scale and fine scale [18].

Similar to the literature [20], we further assume that $\widehat{\mathbf{u}}$ and $\widehat{\mathbf{w}}$, although nonzero within background integral cell $\Omega^{K}$, vanish identically over the $\Omega^{K}$ boundaries when the influence factor $\alpha$ approaches to 1 :

$$
\begin{aligned}
\widehat{\mathbf{u}} & =0, \\
\widehat{\mathbf{w}} & =0 .
\end{aligned}
$$

Substituting (9) into the standard variational form (8), we get

$$
\begin{gathered}
(\nabla(\overline{\mathbf{w}}+\widehat{\mathbf{w}}), \eta \nabla(\overline{\mathbf{u}}+\widehat{\mathbf{u}}))-(\nabla \cdot(\overline{\mathbf{w}}+\widehat{\mathbf{w}}), p)=(\overline{\mathbf{w}}+\widehat{\mathbf{w}}, \mathbf{b}), \\
(q, \nabla \cdot(\overline{\mathbf{u}}+\widehat{\mathbf{u}}))=0 .
\end{gathered}
$$

Next, employing the linearity between coarse scale $\overline{\mathbf{w}}$ and fine scale $\widehat{\mathbf{w}}$, (11) can be split into coarse scale problem and fine scale problem. The coarse scale problem $\bar{W}$ and fine scale problem $\widehat{W}$ can be written as follows:

$$
\begin{gathered}
\bar{W}:(\nabla \overline{\mathbf{w}}, \eta \nabla(\overline{\mathbf{u}}+\widehat{\mathbf{u}}))-(\nabla \cdot \overline{\mathbf{w}}, p)=(\overline{\mathbf{w}}, \mathbf{b}), \\
(q, \nabla \cdot(\overline{\mathbf{u}}+\widehat{\mathbf{u}}))=0, \\
\widehat{W}:(\nabla \widehat{\mathbf{w}}, \eta \nabla(\overline{\mathbf{u}}+\widehat{\mathbf{u}}))-(\nabla \cdot \widehat{\mathbf{w}}, p)=(\widehat{\mathbf{w}}, \mathbf{b}) .
\end{gathered}
$$

Subsequently, we will solve the fine scale problem (14) first to obtain the fine scale solution $\widehat{\mathbf{u}}$ and then substitute it into the coarse fine problems (12) and (13), thereby eliminating the explicit appearance of the fine scale $\widehat{\mathbf{u}}$ while still modeling its effect.
3.3. The Solution of the Fine Scale Problem. In order to obtain fine scale solution $\widehat{\mathbf{u}}$ analytically from $\widehat{W}$, using the bubble function in each $\Omega^{K}$, finally we can obtain the fine scale solution $\widehat{\mathbf{u}}$ over the $\Omega^{K}$ as follows:

$$
\left.\widehat{\mathbf{u}}\right|_{\Omega^{k}}=b_{1}^{K}(\xi) \mathbf{K}^{-1} \mathbf{R},
$$

where

$$
\begin{gathered}
\mathbf{K}=\int_{\Omega^{k}} \eta \nabla b_{1}^{K}: \nabla b_{2}^{K} d \Omega \mathbf{I}+\int_{\Omega^{k}} \eta \nabla b_{1}^{K} \otimes \nabla b_{2}^{K} d \Omega, \\
\mathbf{R}=\int_{\Omega^{k}} b_{2}^{K} \overline{\mathbf{r}} d \Omega, \\
\overline{\mathbf{r}}=\mathbf{F}-\mathbf{u}^{c} \cdot \nabla \overline{\mathbf{u}}+\frac{1}{\operatorname{Re}} \nabla \cdot(\eta \nabla \overline{\mathbf{u}})-\nabla p .
\end{gathered}
$$

Here, $I$ is a $n d \times n d$ identity matrix, both $\nabla b_{1}^{K}$ and $\nabla b_{2}^{K}$ are $n d \times 1$ vectors of gradient of the bubble function, and $n d$ indicates the dimension of the problem.

3.4. The Solution of the Coarse Scale Problem. Once we have obtained the fine scale solution $\widehat{\mathbf{u}}$, substituting it into the coarse fine problem, we get

$$
\begin{aligned}
& (\nabla \overline{\mathbf{w}}, \eta \nabla \overline{\mathbf{u}})-(\nabla \cdot \overline{\mathbf{w}}, p)+(\nabla \cdot(\eta \nabla \overline{\mathbf{w}}), \boldsymbol{\kappa}(-\nabla \cdot(\eta \nabla \overline{\mathbf{u}})+\nabla p)) \\
& \quad=(\overline{\mathbf{w}}, \mathbf{b})+(\nabla \cdot(\eta \nabla \overline{\mathbf{w}}), \boldsymbol{\kappa} \mathbf{b}) \\
& (q, \nabla \cdot \overline{\mathbf{u}})+(\nabla q, \boldsymbol{\kappa}(-\nabla \cdot(\eta \nabla \overline{\mathbf{u}})+\nabla p))=(\nabla q, \boldsymbol{\kappa} \mathbf{b}),
\end{aligned}
$$

where

$$
\begin{aligned}
\boldsymbol{\kappa}= & b_{1}^{K} \int_{\Omega^{k}} b_{2}^{K} d \Omega \\
& \times\left[\int_{\Omega^{k}} \eta \nabla b_{1}^{K}: \nabla b_{2}^{K} d \Omega \mathbf{I}+\int_{\Omega^{k}} \eta \nabla b_{1}^{K} \otimes \nabla b_{2}^{K} d \Omega\right]^{-1} .
\end{aligned}
$$

Compared with the standard variational form (see (8)), it is important to note that the third term on the left has appeared in (17) and the second term on the left appeared in (18) owing to the assumption that there exists fine scale in the problem, which in fact models the effect of the fine scale.

\section{Numerical Examples}

4.1. A Circular Cavity. The flow domain considered here is the unit circle as shown in Figure 1. The upper half of the boundary is assumed to move with a unit tangential velocity in the counterclockwise direction, while the lower half is set with no-slip and impervious Dirichlet conditions. This popular benchmark problem is used to demonstrate whether the algorithm is robust, and the numerical results will be 


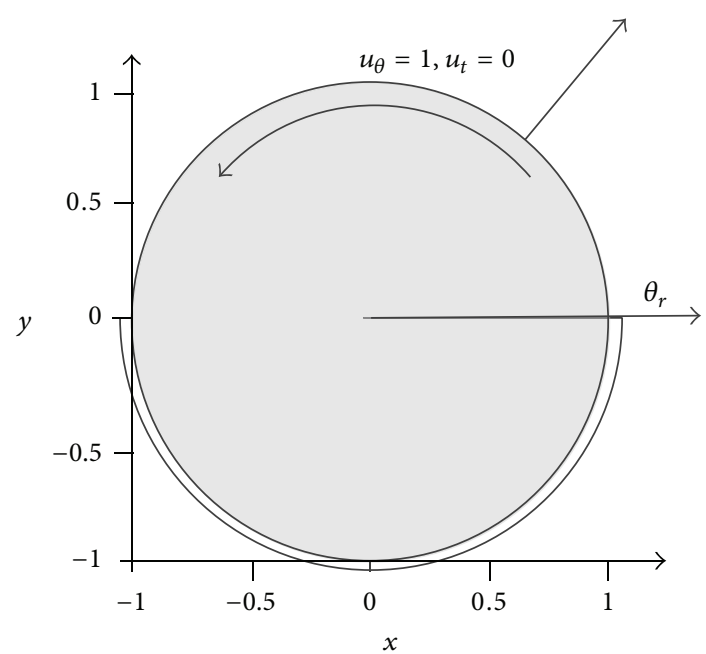

FIGURE 1: Geometry and boundary conditions for a circular cavity flow problem.

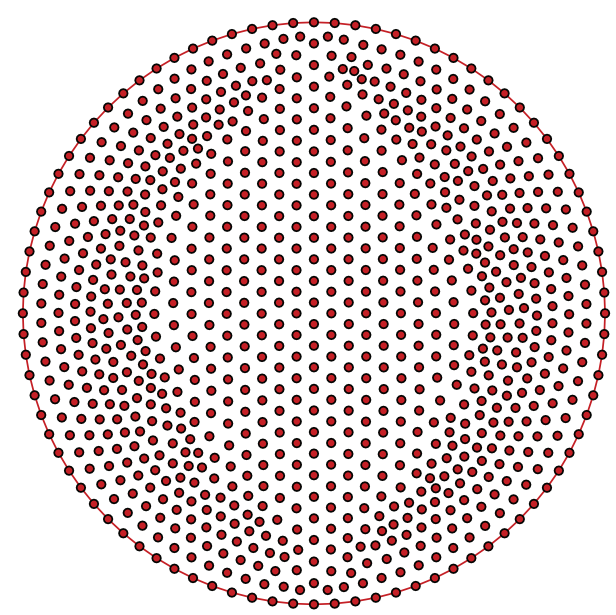

FIGURE 2: 905 irregular nodes distribution for example 1.

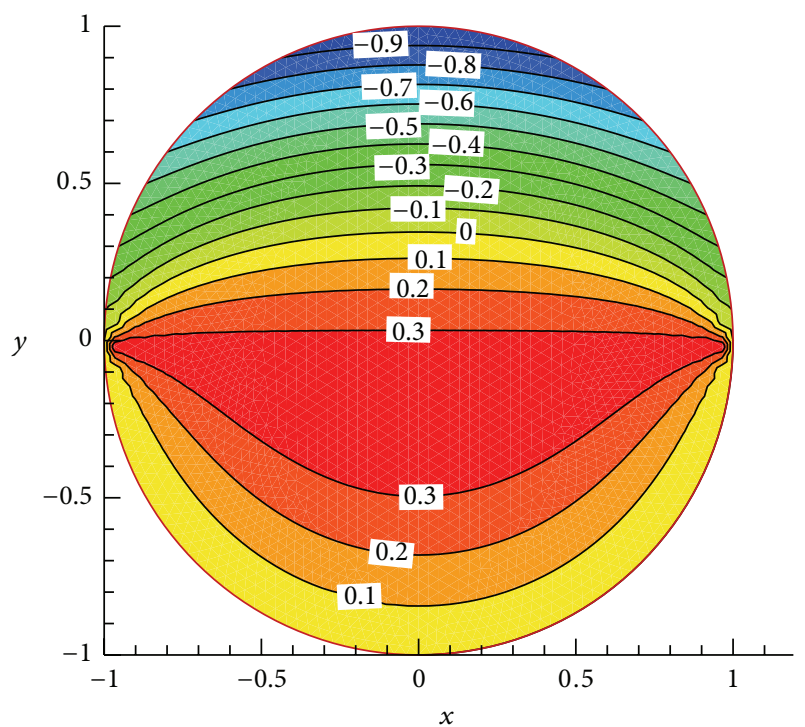

FIGURE 3: $u$-velocity contour for the Stokes flow in a circular cavity.

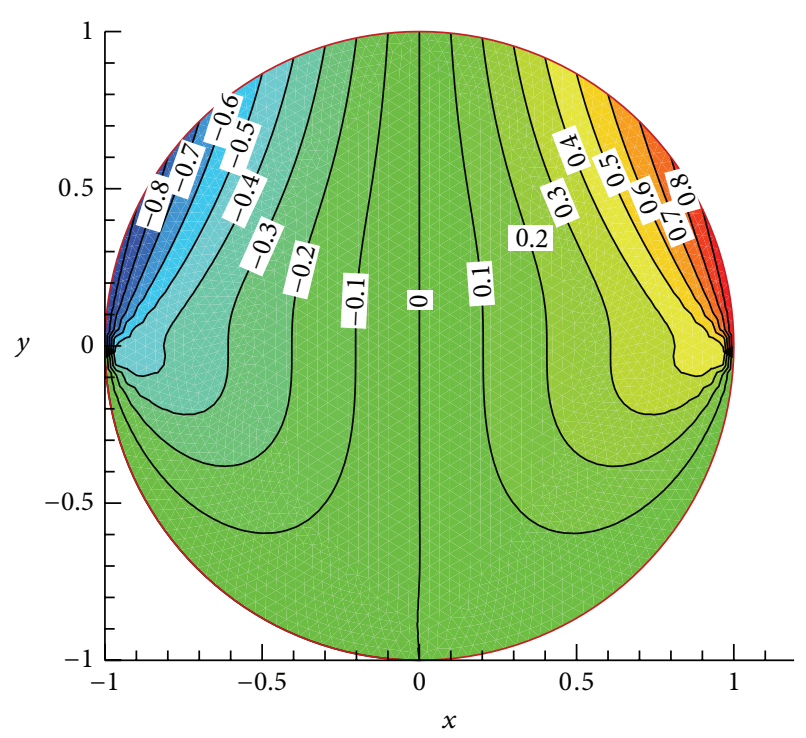

FIGURE 4: $v$-velocity contour for the Stokes flow in a circular cavity.

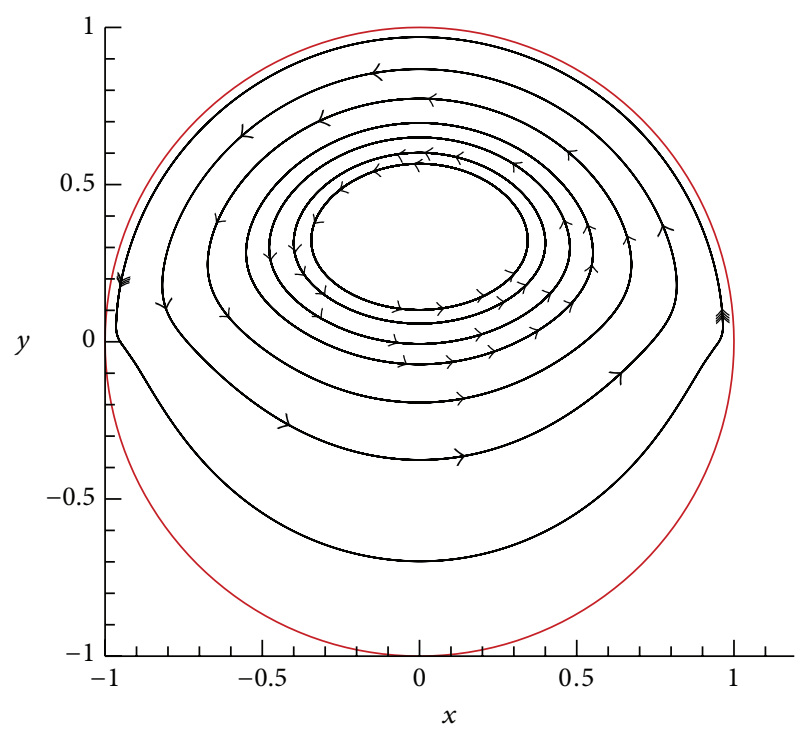

FIGURE 5: Streamline for the Stokes flow in a circular cavity.

compared with the analytical solutions that are given as follows [26, 27]:

$$
\begin{aligned}
u= & -\frac{y}{\pi}\left[\frac{\pi}{2}+a \tan \left(\frac{2 y}{1-x^{2}-y^{2}}\right)\right] \\
& +\frac{\left(1-x^{2}-y^{2}\right)\left(1-x^{2}+y^{2}\right)}{\pi\left[\left(1-x^{2}-y^{2}\right)^{2}+4 y^{2}\right]} \\
v= & \frac{x}{\pi}\left[\frac{\pi}{2}+a \tan \left(\frac{2 y}{1-x^{2}-y^{2}}\right)\right] \\
& -\frac{2 x y\left(1-x^{2}-y^{2}\right)}{\pi\left[\left(1-x^{2}-y^{2}\right)^{2}+4 y^{2}\right]} .
\end{aligned}
$$




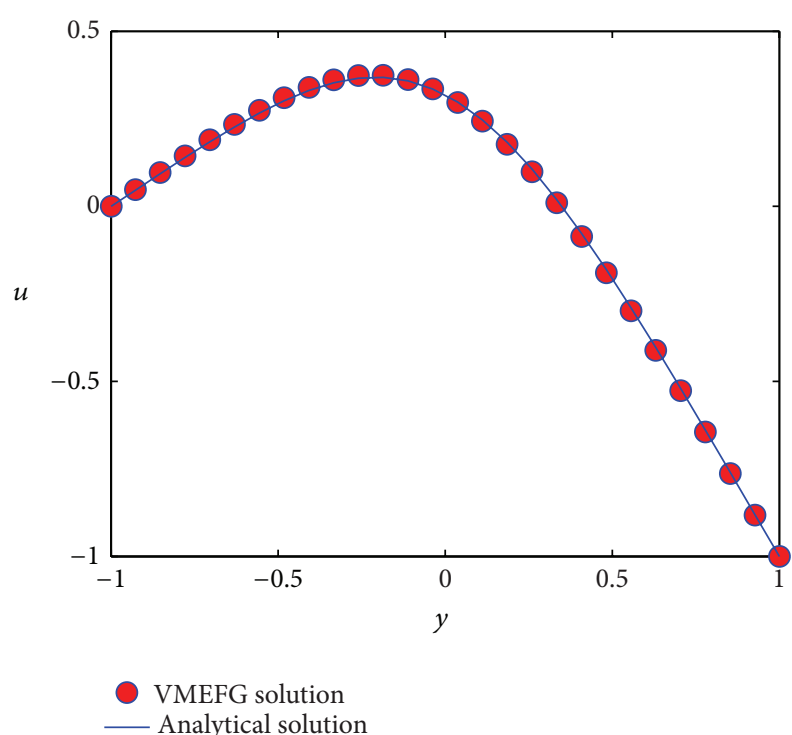

FIGURE 6: Comparison of $u$-velocity profile along $x=0$ obtained by VMEFG method and the analytical solution.

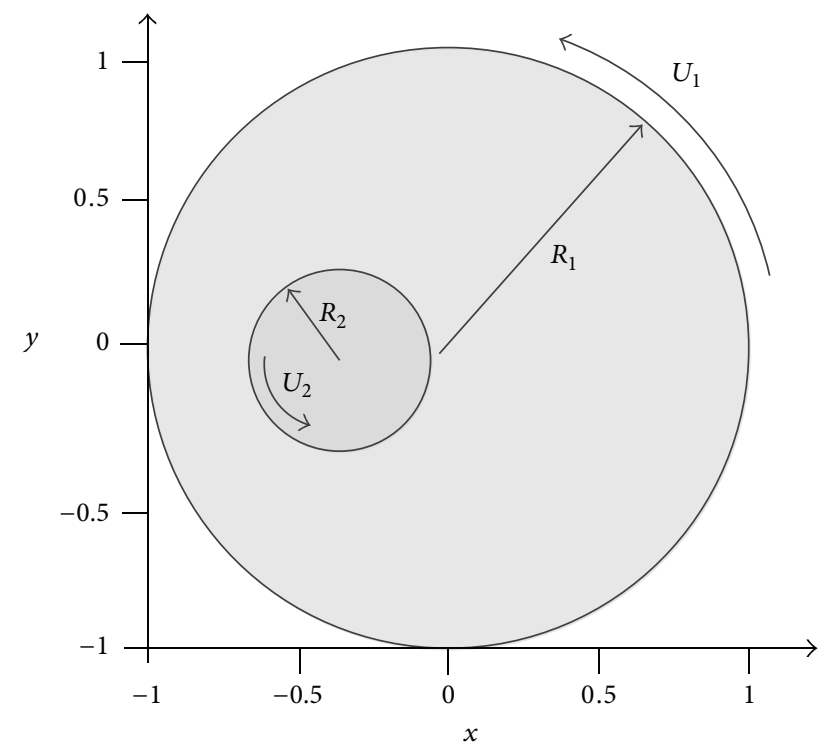

FIGURE 7: Geometry and boundary conditions for Stokes flow between eccentric rotating cylinders.

For this problem, 905 irregular nodes distribution is employed as shown in Figure 2. Due to the fact that VMEFG method needs background mesh to calculate the integral, for example, (17), the triangle background mesh which is similar to the finite element is used in the paper for computational convenience, and seven Gaussian points are used in each triangular region for domain integral. The results obtained by VMEFG are shown as the $u$ - and $v$-velocity contours, as well as streamline in Figures 3, 4, and 5, respectively. It can be seen that the presented results are in excellent agreement with the reported results $[26,27]$. To demonstrate further the accuracy of the VMEFG method, Figure 6 shows the comparison of $u$ velocity profile along the vertical line $x=0$ with the analytical

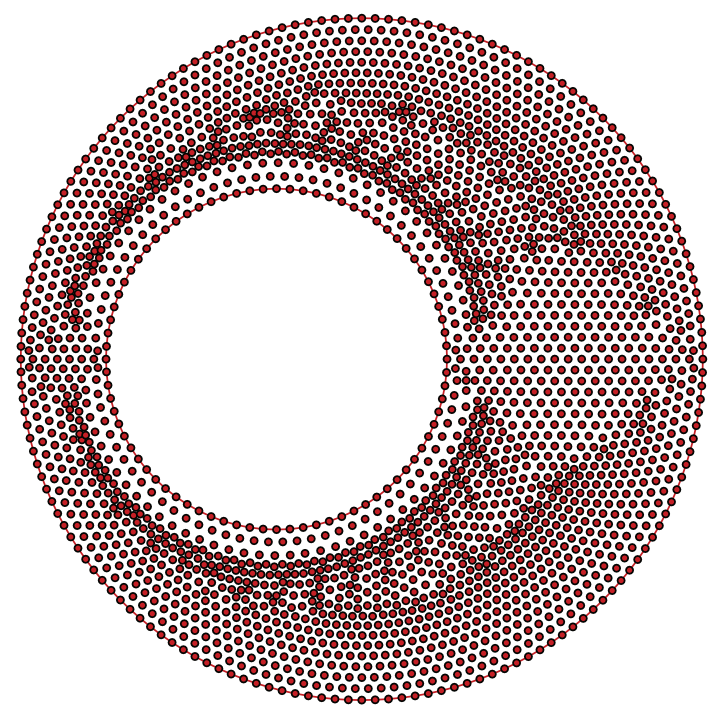

FIGURE 8: 2423 irregular nodes distribution for example 2.

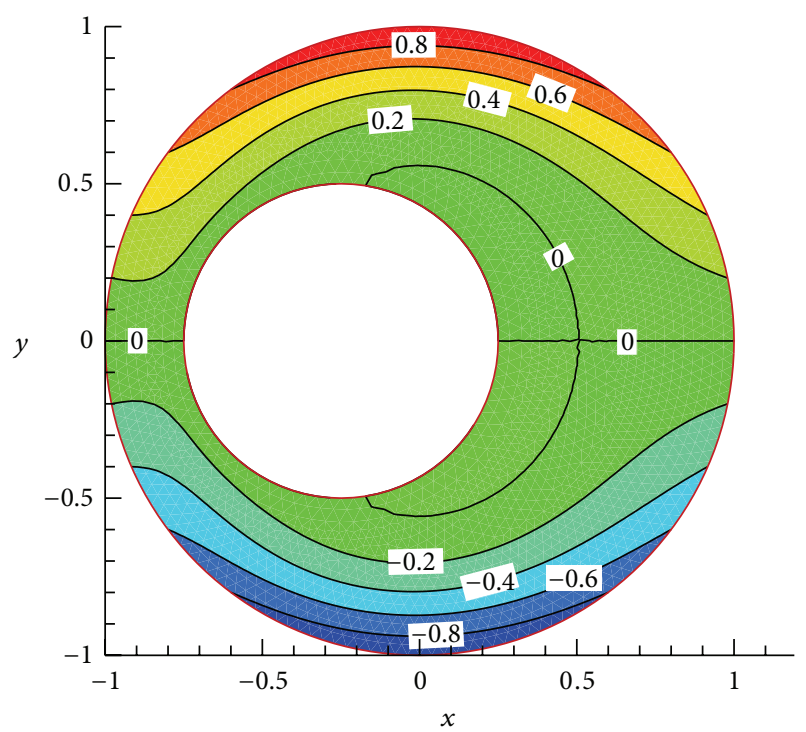

FIGURE 9: $u$-velocity contour for the Stokes flow between eccentric rotating cylinders.

solution. It can be noted that the results are very close to the analytical solution.

4.2. A Circular Cavity with Eccentric Rotating Cylinder. The second example considered here is a Stokes flow confined in the two eccentric cylinders. The geometry and boundary conditions of the problem are sketched in Figure 7. The outer cylinder with radius $R_{1}=1$ centers on the original, and the inner cylinder with $R_{2}=0.5$ has center lying on $(-0.25$, 0 ). No-slip boundary conditions are specified on the fluidsolid interfaces. The parameters $U_{1}$ and $U_{2}$ are defined for the circumferential velocities of the outer and inner cylinders, respectively. In this study, the outer cylinders rotate clockwise and the inner cylinder is kept stationary $\left(U_{1}=-1, U_{2}=0\right)$. 


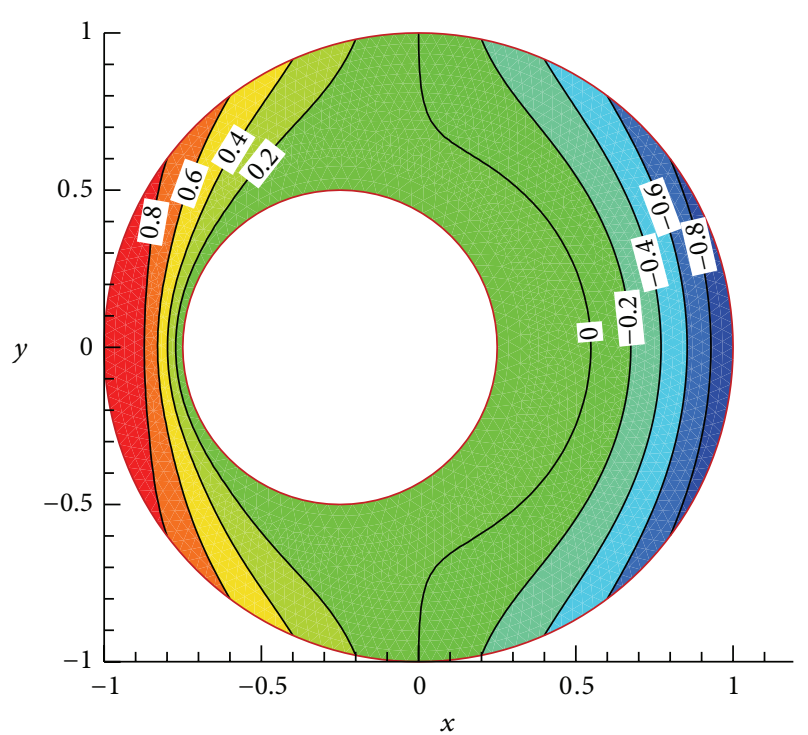

FIGURE 10: $v$-velocity contour for the Stokes flow between eccentric rotating cylinders.

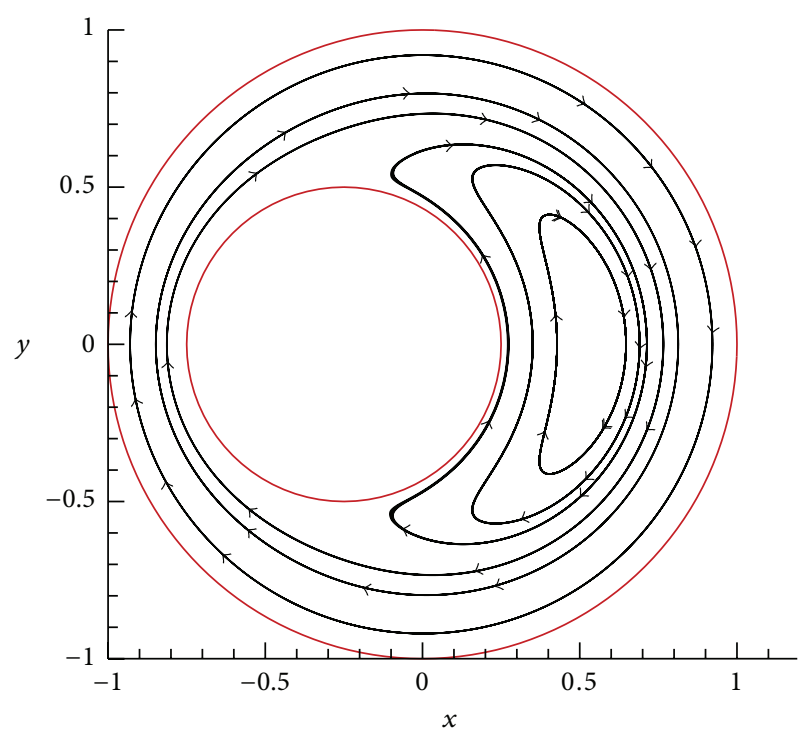

FIGURE 11: Streamline for the Stokes flow between eccentric rotating cylinders.

This problem was also analyzed by a boundary-type meshless method of fundamental solutions (MFS) in [28].

In this study, 2423 nodes are irregularly distributed in the computational geometry as shown in Figure 8. The illustrations on the distributions of $u$ - and $v$-velocity contours, as well as streamline, are shown in Figures 9, 10, and 11. It can be seen that these exhibit the symmetric characteristics of the flow variables as expected for the Stokes flow. To give a more quantitative understanding of accuracy, the comparison of computed $v$-velocity along $y=0$ with the results obtained by MFS is shown in Figure 12. It can be noted that the results obtained by VMEFG are in good agreement with the results by Young et al. [28].

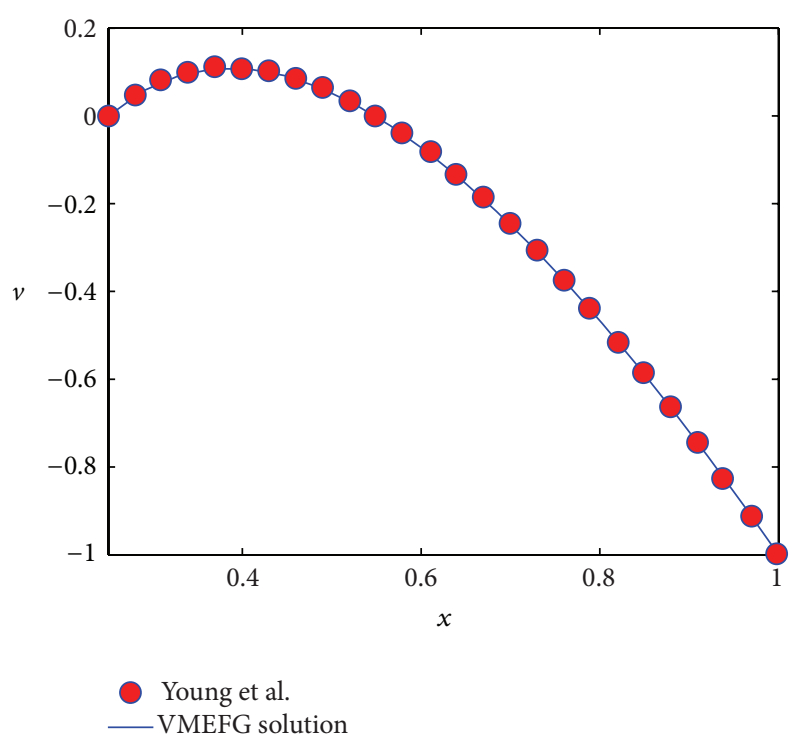

FIGURE 12: Comparison of $v$-velocity profiles along $y=0$ for the eccentric cylinders.

\section{Conclusion}

In the paper, VMEFG method is extended to analyze twodimensional Stokes flow in an irregular geometry. Results are compared with available analytical and other numerical results. And the results show that VMEFG is accurate and robust, even for complex irregular geometry.

\section{Conflict of Interests}

The authors declare that there is no conflict of interests regarding the publication of this paper.

\section{Acknowledgments}

The support from the Natural Sciences Foundation of China (nos. 11102101 and 11171181), the Academic Mainstay Foundation of Hubei Province of China (nos. T201103 and Q20111208), and the Science Foundation of China Three Gorges University (no. KJ2009B058) is fully acknowledged.

\section{References}

[1] T. Belytschko, Y. Krongauz, D. Organ, M. Fleming, and P. Krysl, "Meshless methods: an overview and recent developments," Computer Methods in Applied Mechanics and Engineering, vol. 139, no. 1-4, pp. 3-47, 1996.

[2] V. P. Nguyen, T. Rabczuk, S. Bordas, and M. Duflot, "Meshless methods: a review and computer implementation aspects," Mathematics and Computers in Simulation, vol. 79, no. 3, pp. 763-813, 2008.

[3] G. R. Liu and Y. T. Gu, An Introduction to Meshfree Methods and their Programming, Springer, Amsterdam, The Netherlands, 2005.

[4] G. R. Liu, Mesh Free Methods: Moving Beyond the Finite Element Method, CRC Press, Boca Raton, Fla, USA, 2002. 
[5] S. N. Atluri and S. P. Shen, The Meshless Local Petrov-Galerkin (MLPG) Method, Tech Science Press, Encino, Calif, USA, 2002.

[6] A. Huerta, Y. Vidal, and P. Villon, "Pseudo-divergence-free element free Galerkin method for incompressible fluid flow," Computer Methods in Applied Mechanics and Engineering, vol. 193, no. 12-14, pp. 1119-1136, 2004.

[7] T. P. Fries and H. G. Matthies, "A stabilized and coupled meshfree/meshbased method for the incompressible Navier-Stokes equations. I. STAbilization," Computer Methods in Applied Mechanics and Engineering, vol. 195, no. 44-47, pp. 6205-6224, 2006.

[8] T. Fries and H. G. Matthies, "A stabilized and coupled meshfree/meshbased method for the incompressible Navier-Stokes equations. Part II. Coupling," Computer Methods in Applied Mechanics and Engineering, vol. 195, no. 44-47, pp. 6191-6204, 2006.

[9] H. Lin and S. N. Atluri, "The meshless local Petrov-Galerkin (MLPG) method for solving incompressible Navier-Stokes equations," Computer Modeling in Engineering and Sciences, vol. 2, no. 2, pp. 117-142, 2001.

[10] Q. L. Duan and X. K. Li, "A meshless PSPG formulation for the incompressible Stokes flow," Chinese Journal of Computational Mechanics, vol. 24, no. 2, pp. 192-214, 2007.

[11] X. K. Zhang, K. C. Kwon, and S. K. Youn, "Least-squares meshfree method for incompressible Navier-Stokes problems," International Journal for Numerical Methods in Fluids, vol. 46, no. 3, pp. 263-288, 2004.

[12] Z. C. Xuan and X. K. Zhang, "Simulation of Stokes flow over microelectrodes with least-squares meshfree method," Simulation Modelling Practice and Theory, vol. 16, no. 3, pp. 294314, 2008.

[13] C. You, Y. Qiu, X. Xu, and D. Xu, "Numerical simulations of viscous flows using a meshless method," International Journal for Numerical Methods in Fluids, vol. 58, no. 7, pp. 727-741, 2008.

[14] A. Shamekhi and K. Sadeghy, "On the use of characteristicbased split meshfree method for solving flow problems," International Journal for Numerical Methods in Fluids, vol. 56, no. 10, pp. 1885-1907, 2008.

[15] X. K. Li and Q. L. Duan, "Meshfree iterative stabilized TaylorGalerkin and characteristic-based split (CBS) algorithms for incompressible N-S equations," Computer Methods in Applied Mechanics and Engineering, vol. 195, no. 44-47, pp. 6125-6145, 2006.

[16] E. Oñate, S. Idelsohn, O. C. Zienkiewicz, and R. L. Taylor, "A finite point method in computational mechanics. Applications to convective transport and fluid flow," International Journal for Numerical Methods in Engineering, vol. 39, no. 22, pp. 38393886, 1996.

[17] T. P. Fries and H. G. Matthies, "A review of Petrov-Galerkin stabilization approaches and an extension to meshfree methods," http://digisrv-1.biblio.etc.tu-bs.de:8080/docportal/servlets/ MCRFileNodeServlet/DocPortal_derivate_00001549/Document.pdf;jsessionid=13366DF5A0AD985438FA12988E5D79A4.

[18] A. Masud and R. A. Khurram, "A multiscale finite element method for the incompressible Navier-Stokes equations," Computer Methods in Applied Mechanics and Engineering, vol. 195, no. 13-16, pp. 1750-1777, 2006.

[19] L. Zhang, J. Ouyang, X. Zhang, and W. Zhang, "On a multiscale element-free Galerkin method for the Stokes problem," Applied Mathematics and Computation, vol. 203, no. 2, pp. 745753, 2008.
[20] L. Zhang, J. Ouyang, T. Jiang, and C. Ruan, "Variational multiscale element free Galerkin method for the water wave problems," Journal of Computational Physics, vol. 230, no. 12, pp. 5045-5060, 2011.

[21] L. Zhang, J. Ouyang, and X. H. Zhang, "The variational multiscale element free Galerkin method for MHD flows at high Hartmann numbers," Computer Physics Communications, vol. 184, no. 4, pp. 1106-1118, 2013.

[22] S. Soleimani Kutanaei, N. Roshan, A. Vosoughi, A. Barari, and S. Soleimani, "Numerical solution of stokes flow in a circular cavity using mesh-free local RBF-DQ," Engineering Analysis with Boundary Elements, vol. 36, no. 5, pp. 633-638, 2012.

[23] N. Zhao and H. Ren, "The interpolating element-free Galerkin method for 2D transient heat conduction problems," Mathematical Problems in Engineering, vol. 2014, Article ID 712834, 9 pages, 2014.

[24] X. Zhuang and Y. Cai, "A meshless local Petrov-Galerkin Shepard and least-squares method based on duo nodal supports," Mathematical Problems in Engineering, vol. 2014, Article ID 806142, 11 pages, 2014.

[25] X. H. Zhang, P. Zhang, and L. Zhang, "An improved meshless method with almost interpolation property for isotropic heat conduction problems," Engineering Analysis with Boundary Elements, vol. 37, no. 5, pp. 850-859, 2013.

[26] F. Tan, Y. Zhang, and Y. Li, "Development of a meshless hybrid boundary node method for Stokes flows," Engineering Analysis with Boundary Elements, vol. 37, no. 6, pp. 899-908, 2013.

[27] X. Li, "Meshless analysis of two-dimensional Stokes flows with the Galerkin boundary node method," Engineering Analysis with Boundary Elements, vol. 34, no. 1, pp. 79-91, 2010.

[28] D. L. Young, C. L. Chiu, C. M. Fan, C. C. Tsai, and Y. C. Lin, "Method of fundamental solutions for multidimensional Stokes equations by the dual-potential formulation," European Journal of Mechanics B: Fluids, vol. 25, no. 6, pp. 877-893, 2006. 


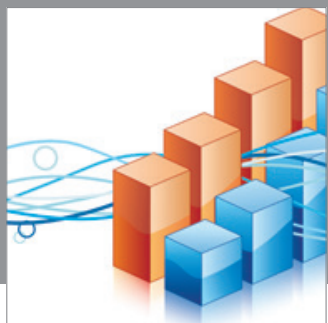

Advances in

Operations Research

mansans

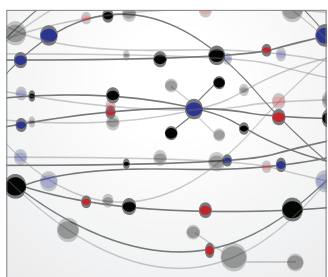

The Scientific World Journal
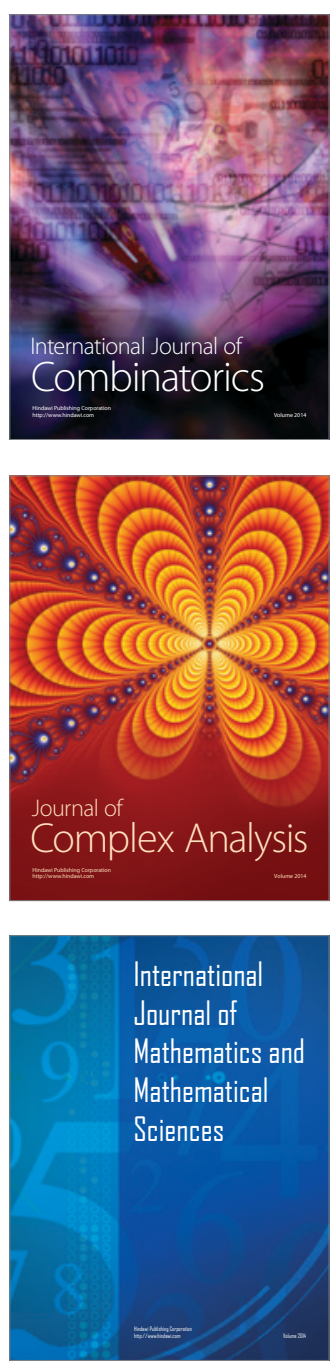
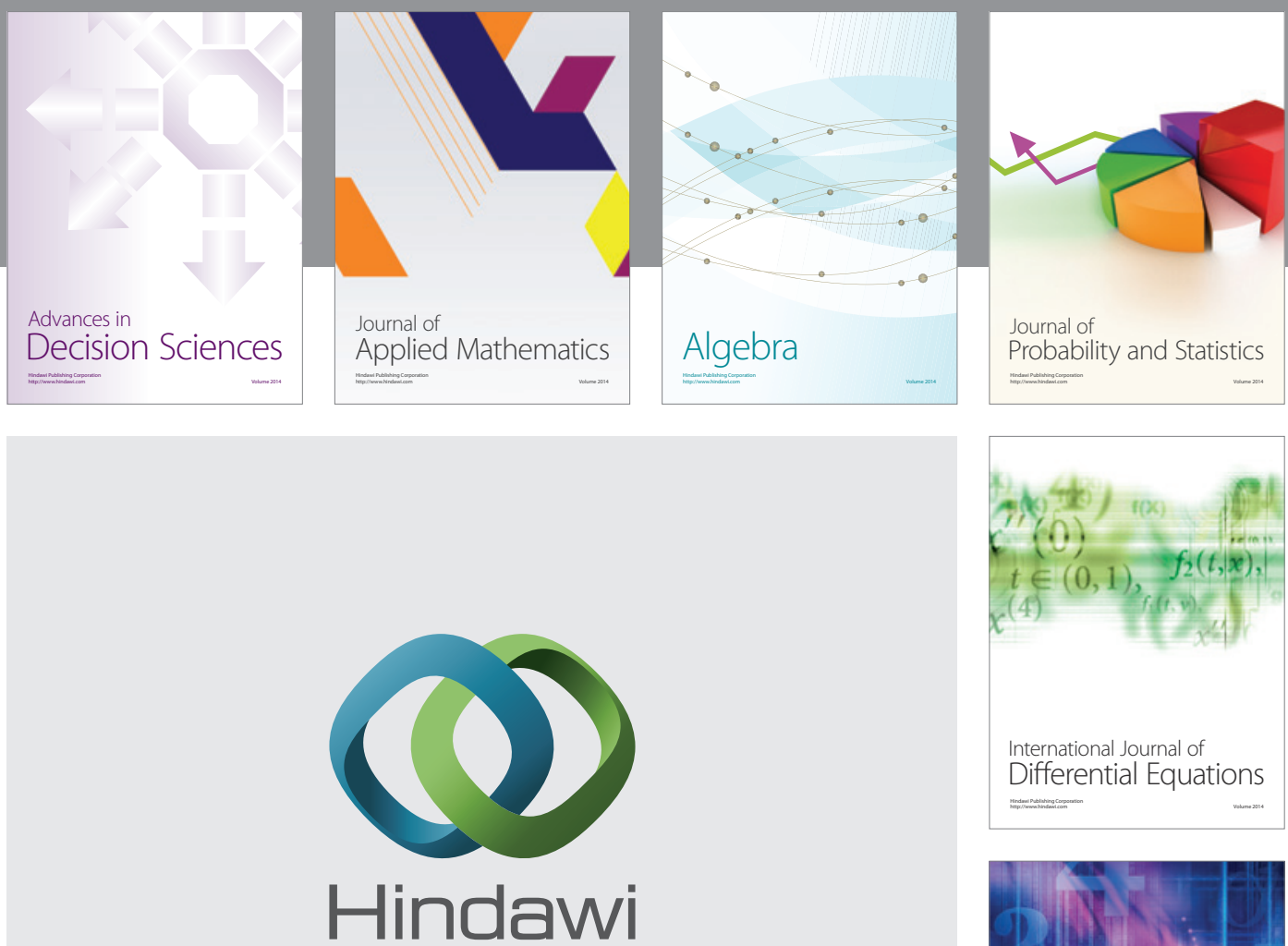

Submit your manuscripts at http://www.hindawi.com
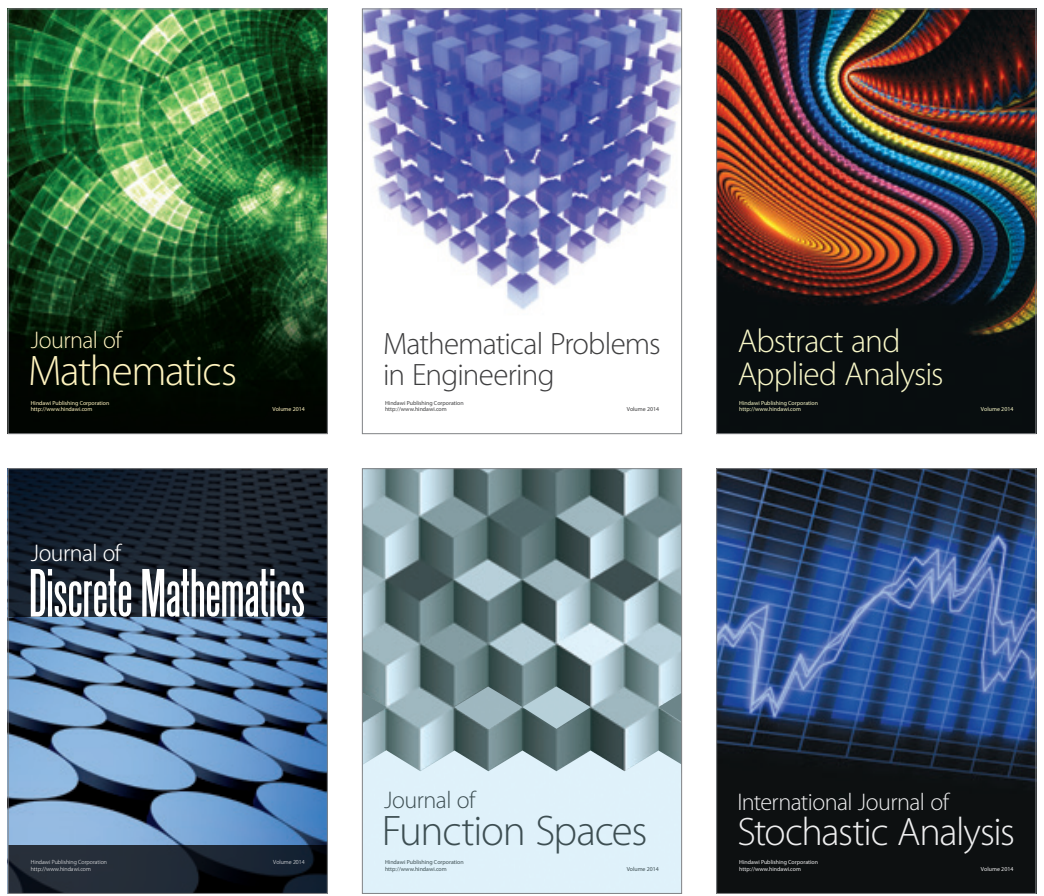

Journal of

Function Spaces

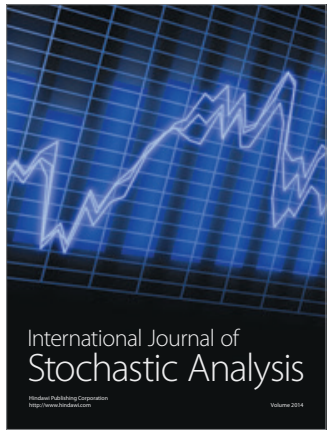

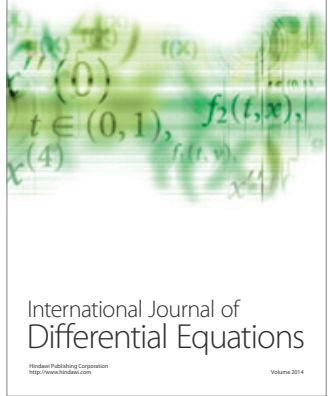
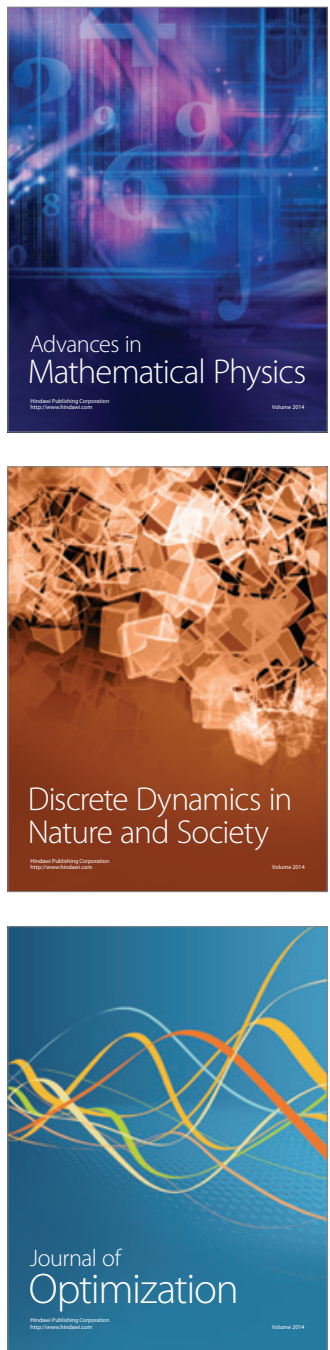